Culture et histoire dans l'espace roman

Crise(s) dans le monde ibérique et ibéro-américain

\title{
Crise de lecture?
}

\section{Pénélope Laurent}

\section{(2) OpenEdition}

\section{Journals}

Édition électronique

URL : https://journals.openedition.org/cher/3607

DOI : $10.4000 /$ cher.3607

ISSN : 2803-5992

\section{Éditeur}

Presses universitaires de Strasbourg

\section{Édition imprimée}

Date de publication : 1 décembre 2015

Pagination : 93-103

ISBN : 978-2-86820-913-9

ISSN : 1968-035X

\section{Référence électronique}

Pénélope Laurent, «Crise de lecture ? », reCHERches [En ligne], 15 | 2015, mis en ligne le 01 décembre 2021, consulté le 15 décembre 2021. URL : http://journals.openedition.org/cher/3607 ; DOI : https:// doi.org/10.4000/cher.3607

\section{(ब) $(0 \bigcirc$}

Ce(tte) œuvre est mise à disposition selon les termes de la Licence Creative Commons Attribution Pas d'Utilisation Commerciale - Partage dans les Mêmes Conditions 4.0 International. 


\title{
Crise de lecture?
}

\author{
Pénélope Laurent \\ Université de Perpignan - Via Domitia
}

\begin{abstract}
$\mathrm{C}$ rise économique, crise financière, crise pétrolière, crise politique, crise diplomatique, crise environnementale, crise morale, crise existentielle, qui ou qu'est-ce qui ne souffre pas d'une crise aujourd'hui? Qui oserait remettre en question ce qui semble, de toute évidence, le concept supposément opérant pour décrire notre époque postmoderne? La postmodernité accepte diverses définitions, généralement reliées à la déconstruction, que l'on pense à Jacques Derrida ou encore à Jean-François Lyotard et à la fin des grands récits. Toutes ses définitions semblent faire d'elle un moment critique, un moment de crise. Mais cette récurrence critique, à force de répéter l'avènement d'une crise - de la crise -, ne nous donne-t-elle pas l'impression quelle désire la crise plus qu'elle ne la rejette? La crise ne serait-elle pas plutôt une intention, pas toujours assumée, qu'une réalité dans la mesure où nous ne parvenons pas à en sortir? D’une façon paradoxale, la postmodernité semble prendre plaisir à parler de crise sans jamais parvenir à en produire - telle la Révolution à laquelle elle a cessé de croire, celle qui avance sans possibilité de retour -, embourbée un peu malgré elle dans le collage, le recyclage, le "présentisme».

Je propose d'interroger ici la notion de "crise de la représentation», si souvent appliquée par la critique à la narration contemporaine, ce qui m’amènera à revenir sur la définition de la représentation. J'utiliserai l'idée de crise du régime moderne d'historicité telle que la définit François Hartog dans Régimes d'historicité et tenterai de la mettre en perspective avec la représentation. Le court roman du guatémaltèque Rodrigo Rey Rosa, Severina (2011) nous donne l'occasion de penser la représentation et sa supposée crise à travers son personnage éponyme, l'énigmatique voleuse de livres et de cœurs. L'amour et les livres sous diverses déclinaisons - l'amour pour les livres, des livres pour être aimée - sont particulièrement propices à une réflexion sur les relations entre un lecteur ou une lectrice et la littérature, narrative en particulier. Mais si Severina
\end{abstract}


semble osciller entre hystérie et cleptomanie, les crises de Madame Bovary nous paraissent bien lointaines en ce $\mathrm{XxI}^{\mathrm{e}}$ siècle naissant.

\section{La lectrice, un personnage en crise?}

Severina, le personnage qui donne son titre au roman de Rey Rosa, est une voleuse de livres qui séduit le personnage-narrateur, libraire qui vient de se séparer depuis peu, sans que l'on ne comprenne bien si son jeu est intentionnel ou non, si la séduction fait partie ou non d'une stratégie pour voler toujours plus de livres o si le vol de livres est lui-même la stratégie de séduction qu’elle adopte, avec lui comme avec d’autres. Ces deux activités sont étroitement liées à la diégèse, du début à la fin de ce court roman. Severina est énigmatique, Severina ne s'appelle pas Severina, peut-être s'appelle-t-elle Ana, peut-être est-elle mariée à un vieil homme, peut-être que celui-ci n'est autre que son père, peut-être même son grand-père, sans doute est-elle uruguayenne ou argentine, à moins qu'elle ne soit hondurienne ou italienne. Son destin est peut-être celui de vivre des livres et des libraires, et le leur d'être assassinés par elle, comme l'insinue la fin du roman. On ne sait pas. Severina est un personnage mystérieux qui échappe autant au personnage-narrateur qu'au lecteur. C'est un être assez irréel, le personnage de papier qui vole des livres, c'est-à-dire in fine, du papier.

L'intrigue est assez mince et se construit autour de la liaison de la lectrice et du libraire comme s'il s'agissait d'un roman policier: le personnage-narrateur, jamais nommé mais jouissant d'un peu plus d'épaisseur psychologique que sa voleuse de livres - puisqu'il est narrateur et s'exprime à la première personne - confie au lecteur comment il est tombé amoureux de la belle Ana Severina, comment il a tenté d'en être aimé de retour et comment il a fini par laisser son propre destin entre ses mains - de cleptomane. Au bout des cent pages, il ferme le livre par une mention en italique "Ciudad de Guatemala, 31 de diciembre, 2009» (Rey Rosa 2012: 104) après avoir envisagé dêtre assassiné des mains de sa bien-aimée. Cette œuvre, qu'il faut bien qualifier d'ouverte, a toutefois un caractère cyclique que l'on peut remarquer tant à travars cette date - le dernier jour de l'année - quà travars l'année - le chiffre neuf symbolisant tout à la fois un cycle qui se ferme et la promesse de celui à venir. Ce récit est présenté au lecteur comme un récit écrit, un témoignage (fictif). Mais un témoignage qui laisse son lecteur en suspens jusqu’à la fin parce que nous ignorons si le personnage-narrateur va mourir ou non, pourquoi Severina agit de la sorte et comment termine cette histoire policière de l'amour consommé. Et la mention finale de Ciudad de Guatemala ajoute au mystère puisque les deux amants sont censés sêtre envolés ensemble à Mexico. Que s'est-il passé? Sont-ils revenus? Le témoignage semble faire appel à un complément d'information (entre le temps raconté et le présent d'énonciation) que le lecteur devrait avoir mais qu'il n’a pas. Ou tout cela na-t-il été qu'une illusion, comme se le demande le narrateur auquel le lecteur s'identifie, au moins partiellement? («un engaño, pero no un engaño llevado a cabo por dos seres humanos para burlar a otro sino un desvarío de 
mi propia imaginación») (Rey Rosa 2012:93). S’agirait-il d’un délire de notre imagination? Si le narrateur ne s'inquiète pas tellement d’offrir au lecteur un récit qui ne finit pas, le lecteur en revanche aimerait bien comprendre cette partition jouée à deux, quatre ou six mains.

Severina joue avec l'oubli et les «blancs» du texte. La citation en épigraphe de William Carlos William, "What power has love but forgiveness?» évoque l'aveuglement de celui ou celle qui tombe amoureux(se). La mention du titre du recueil Asphodel, That Greeny Flower ajoute une dimension tragique puisque l'asphodèle est la fleur des morts - que l'on plaçait sur les tombes dans la Grèce Antique - et de l'Hadès dans la mythologie grecque - le pré d'Asphodèle étant le lieu où séjournaient les morts n'ayant commis, précisément, aucun crime. Or, Ana Severina enterre son énigmatique père-grand-père-amant après l'avoir euthanasié, et le narrateur a bien peur qu'il ne lui arrive la même chose à la fin du roman. Au-delà de l'interprétation symbolique (de fin du roman/fin d'une histoire d'amour), il faut penser que dans le roman l'oubli est la condition de la multiplicité actantielle: pour vivre plusieurs vies, Ana Severina a besoin d'oublier. Et lorsque le narrateur lui demande de lui raconter sa vie, Ana Severina répond mystérieusement: "Tenemos muchas vidas» (Rey Rosa 2012:97). Elle et son grand-père ont des faux-papiers. Leur identité est flottante, changeante. Le grand-père, comme Ana Severina, change d'accent lorsqu'il parle et le narrateur remarque que son aspect physique se modifie d'un jour à l'autre. Et le nom de famille de ce grand-père, qui est aussi peut-être un père ou un amant, s'avère être Blanco, de façon ironique. Otto Blanco. Blanc, aussi blanc qu'une page blanche, comme la promesse d'une nouvelle vie possible pour Ana Severina avec laquelle il a, peut-être, contracté "un mariage blanc» comme l'évoque le narrateur (Rey Rosa 2012:52). Et le narrateur lui-même finira, à la fin du roman, par acquérir un faux passeport et, par contrecoup, le nom Blanco. L'oubli assaille le présent, effaçant les traces du passé et rendant plus difficile la projection vers l'avenir.

Ce halo de mystère qui semble envahir le roman peut être associé à deux types de crise: la crise de la représentation - dont la désignation me semble inexacte - et celle du régime moderne d'historicité, le présentisme.

\section{Crise de la lecture?}

Commençons par la crise de la représentation esthétique, en tant que phénomène et notion, déjà bien étudiée. La représentation est la capacité de re-présenter, de présenter une seconde fois, à distance, grâce à une nécessaire coupure sémiotique (le signe nétant pas la chose, le mot chien ne mord pas). En littérature, d'une certaine façon, la distance est double si nous la comparons à d'autres types de représentations comme, par exemple, la presse ou la photographie testimoniale, parce que la fiction agit comme un filtre plus fort. Et malgré tout, nombreux sont ceux qui continuent de confondre narrateur et écrivain, la réalité de la fiction et la réalité alors que la première s'inclut dans la seconde sans nécessairement la refléter. L'univers fictionnel dispose de sa propre autonomie, 
celle d'un système auto-organisateur ouvert (Ezquerro 2002). La représentation par la fiction se nourrit de la réalité, faite de représentations, exprime des réalités mais ne reflète pas simplement la réalité. La coupure sémiotique crée une distance essentielle et l'aspect différé de la lecture renforce ou duplique cette distance. Cette conjonction de la distance et du différé pourrait être ce que Derrida nomme la «différance». Mais si la distance de la représentation se produit toujours in absentia, cela ne signifie pas qu'il n'y ait pas une présence d'un autre ordre; c'est ainsi que l'entend le philosophe Daniel Bougnoux, le préfixe rede représentation pouvant signifier à la fois «présenter une seconde fois » et «être présent » de façon emphatique. Et nous savons bien que l'expérience de la lecture est un mélange d'ilusio et de distance, d’adhérence et de décollement (Jauss 1978), très plaisant. Cette demande d'un double mouvement de lecture est de plus en plus présente dans la littérature contemporaine et particulièrement dans celle de la postmodernité qui aurait fait l'expérience de la crise de la représentation. La critique littéraire, dont l'étymologie n'est autre que celle du mot crise, n'est pas étrangère à ce processus parce que la littérature postmoderne inclut ou amplifie souvent une critique du mode de représentation en jeu.

Ladite crise de la représentation intervient généralement lorsque la réalité fait irruption et déplace le signe, lorsque celui-ci doit être changé par un autre, considéré alors comme plus réel, plus proche du réel. La crise de la représentation est multiple et prend des formes différentes selon les arts, mais on peut dire quelle se définit peu ou prou par une méfiance vis-à-vis des langages (littéraire, pictural, musical, théâtral, etc.) et de leur capacité à représenter ingénument le réel. Cela se traduit par une plus grande distance: l'intrigue devient moins linéaire et moins complète, elle s'amoindrit, les personnages exhibent des parts d'ombre ou des invraisemblances; la musique utilise les fragments que sont les samples et la répétition au détriment de la mélodie ou de l'harmonie; la peinture cesse dêtre figurative pour se tourner vers l'abstraction; etc. Dans la littérature moderne, l'un des motifs récurrents consiste à introduire des scènes de lecture ou des figures de lecteurs, comme l'analyse Nora Catelli dans Testimonios tangibles. Elle remarque la multiplication de ces scènes et figures au XIX ${ }^{e}$ siècle puis leur disparition au siècle suivant, les romans produisant alors des images qui ne sont en rien représentatives de leur époque. Effectivement, au XIX ${ }^{e}$ siècle les analphabètes, et les femmes en particulier, sont particulièrement nombreux alors que les personnages de lectrices (et les scènes de lecture) sont de plus en plus présents dans la littérature. Et le mouvement s'inverse au $\mathrm{Xx}^{\mathrm{e}}$ siècle: si les lecteurs «réels», et parmi eux les lectrices en particulier, voient leur nombre augmenter avec la massification de l'éducation scolaire, le motif, lui, aurait tendance à disparaitre. Nora Catelli en tire la conclusion que la crise de la représentation est provoquée par ce décalage tandis qu'un nouveau motif s'impose dans la littérature contemporaine, celui de l'objet-livre.

Cette analyse est très convaincante, pertinente à bien des égards et copieusement documentée mais je n'en tire pas la même conclusion : il me semble que le véritable changement (qui n'est pas une crise à proprement parler), c'est 
que la littérature contemporaine introduit non pas un motif mais un dispositif de lecture qui demande au lecteur une attention et une activité de plus en plus importante - et plaisante -, entre l'incrédulité et sa suspension, entre illusion et sens critique. Mais bien sûr Cervantès, les Romantiques d'Iena, Coleridge et Diderot le thématisaient et le plaçaient aussi au cœur de leur œuvre. Ce jeu si savoureux, et si présent dans la littérature contemporaine, est aussi celui de l'ironie romantique. En ce sens, Borges serait l'écrivain paradigmatique de notre postmodernité (lui qui a été si souvent qualifié de "dernier moderne»). La crise de la représentation, avons-nous dit, intervient généralement lorsque la réalité fait irruption et déplace le signe, mais nous pourrions nous demander si ce n'est pas plutôt le contraire, c'est-à-dire lorsque le signe fait irruption et déplace le réel, comme cela a lieu de façon humoristique mais aussi préoccupante dans «Del rigor de la ciencia»: la carte recouvre le territoire, les habitants errent, désorientés, quand la représentation prend les dimensions de la réalité. La représentation, avec sa coupure sémiotique et sa distance, est indispensable car, sans elle, la carte deviendrait le territoire et aurait lieu la véritable crise de la représentation, une crise proprement fantastique.

Aussi la crise de la représentation me semble-t-elle être une désignation abusive. Effectivement, le mot crise provient du langage médical et s'emploie alors que deux solutions peuvent être envisagées, incompatibles entre elles: la guérison ou la mort. Or il n'y a non seulement aucune guérison et aucune mort possible (sauf symbolique) dans le cas de la représentation mais il n'y a pas non plus de maladie ou de «mal»-même s'il est vrai que le motif de la lecture comme maladie est très présent dans la littérature romanesque, avec le Quichotte et Madame Bovary en tête. La représentation n'a jamais fonctionné autrement que par changements à l'intérieur d'une continuité. La crise, en revanche, suppose un changement radical, essentiel, et non pas relatif, dans un environnement ou dans un système dont on présupposerait qu'il devrait être stable. Si on écarte ce présupposé de stabilité, peut-être pourrions-nous envisager les changements de paradigmes dans une plus grande plasticité. La crise débouche, dans le meilleur des cas, sur une sortie de crise. Mais nous ne sortons, jamais, de la représentation, nous ne pouvons pas y échapper.

Toutefois il est vrai que, d'un point de vue historique, la représentation révèle des points d'inflexion certains, et dans la postmodernité notamment, qui est de plus en plus "présentiste » : on délaisse le journalisme pour l'actualité, la littérature semble moins passionner le public que la téléréalité, la mémoire et le patrimoine paraissent remplacer l'Histoire. Et bien souvent lorsque la représentation est aujourd'hui utilisée, c'est pour l'instrumentaliser à des fins économiques ou politiques, comme l'a démontré Christian Salmon avec ses recherches portant sur le storytelling. Pour certains historiens actuels, notre rapport au temps, l'expérience faite par notre époque, postmoderne, qui coïncide avec la fin des grands récits, changent et débouchent sur le présentisme, néologisme créé à partir de la catégorie futurisme. C'est l'hypothèse de François Hartog, qui conçoit et utilise l'instrument heuristique de «régime d'historicité» pour élaborer son 
idée et former des hypothèses sur la façon dont une époque articule le présent à son passé et à son futur. La Révolution française est lévénement qui a marqué une rupture, une véritable crise, dans la conception de l'articulation du passé, du présent et du futur: le régime moderne d'historicité crée une nouvelle tension entre le champ de l'expérience et l'horizon d'attentes, tension qui cesse de voir dans le passé un modèle - le modèle - et projette le moteur du sens de l'Histoire dans le futur. Lautre crise aurait eu lieu deux siècles plus tard, symboliquement en 1989, marquant alors la fin des grands récits (de lémancipation et de l'esprit, selon Lyotard) et des idéologies. Notre époque présentiste donne parfois l'impression de vivre dans un présent perpétuel car la distance entre le champ d'expérience et l'horizon d'attentes est maximale, selon Hartog, à la limite de la rupture. Le passé semble se borner désormais à la mémoire et au patrimoine (en opposition à l'Histoire) tandis que le futur ne nous apparaît que sous la forme d'une crise permanente, d'une menace, dans la mesure où nous ne pouvons plus croire aveuglément au progrès.

C'est lère du soupçon, pour reprendre le titre de l'essai de Nathalie Sarraute, et nous voyons bien que ladite crise de la représentation accompagne un phénomène plus large qui ressemble à la crise du régime moderne d'historicité, au présentisme. Dans cette ère du soupçon, les personnages ne se laissent pas définir entièrement mais exhibent des parts d’ombre, leur indétermination, leurs contradictions; leurs attributs ne sont pas de simples reflets de la réalité; l'intrigue est réduite, se complexifie ou prend de multiples orientations; les points de vue se multiplient pour détrôner le statut de la vérité, etc. Lère du soupçon stimule le lecteur attentif et actif, dans le présent de sa lecture, l'invite à procéder à des relectures, aux va-et-vient constants de la lecture rétrospective et prospective, comme l'explique Wolfgang Iser; tandis qu'une lecture superficielle, linéaire, régie par les «habitudes commerciales et idéologiques de notre société qui recommande de "jeter" l'histoire une fois qu’elle a été consommée ("dévorée") 》 (Barthes 1970:20) est présentiste. La relecture, lente, critique et active, essaie, sans doute en vain, de freiner le présentisme, démêlant la façon dont chaque texte élabore un tissu de sens. Le développement de la critique littéraire et de la théorie de la lecture est lié à la crise de la représentation, puisque la création et la critique s'influencent désormais indéniablement. Le lecteur actif, qui prend une part active dans l'élaboration des sens du texte, tire profit des «blancs» du texte en les investissant de représentations personnelles et par son interprétation subjective. En jouant sur le prénom de notre lectrice Severina, on peut dire qu'un certain type de lecteurs (et de lectrices) devient de plus en plus sévère à l'heure de la massification de la lecture et de la consommation des livres.

Pour en revenir au roman Severina, j’admets volontiers que le lien entre la crise de la représentation et la crise du présentisme d'une part et le roman de Rey Rosa d'autre part ne soit pas évident. En mettant en scène une fille ou petitefille symbolique de Madame Bovary, lectrice sentimentale qui s'identifie aussi à ce qu'elle lit tout en prenant sa revanche sur les libraires, ce roman minimaliste rend compte de deux phénomènes critiques ayant trait à la représentation 
littéraire. Le personnage de Severina est typiquement un personnage effacé et incomplet. L'intrigue, réduite, reprend les codes du roman de suspense sans les mener à leur terme - l'énigme nétant jamais résolue -, jouant avec les attentes et la participation active du lecteur, entre l'identification à l'histoire racontée et la frustration de ne pas la voir complétée. Les personnages et l'intrigue sont les deux éléments de composition essentiels de la littérature narrative moderne et, dans notre cas, la déconstruction les affecte, ouvrant l'espace d'interprétation et d'imagination au lecteur. L'ouvre ouverte, qui assume sa part d'indétermination, fait une place au lecteur, sorte de contrepoint au roman réaliste du XIX ${ }^{e}$ siècle dont l'univers semble désormais trop plein et homogène au lecteur contemporain (Georges Poulet 1979). Severina nest pas Madame Bovary, la construction de ces deux personnages est extrêmement différente: Emma Bovary est un être bien défini, par son environnement social, son histoire familiale, ses caractéristiques physiques et morales, etc. tandis qu'Ana Severina est très peu déterminée, elle se targue d'avoir plusieurs vies et n’a pas d'identité fixe. Mais Madame Bovary agit sur elle de façon paradoxale comme contrepoint. Cette présence paradoxale du contre-modèle semble être paradigmatique de la création postmoderne: le collage, la référence, le recyclage, le fragment proviennent de crises mais semblent incapables de produire une véritable crise. Si Madame Bovary entrait en rupture avec l'horizon d'attentes de son époque, aucun roman ne semble être en mesure, aujourd'hui, de produire une rupture profonde, une crise, une transgression authentique. Les textes littéraires semblent assumer que leur horizon est le lecteur et prévoient pour lui une activité plus ou moins importante en fonction de leur rapport à la fragmentation.

L'ironie romantique est sans doute le marqueur d'une littérature qui a introduit, à défaut d'une crise de la représentation, du moins une critique de la représentation réaliste, mimétique, de la littérature. De fait, le personnagenarrateur non nommé, sous le charme de Severina, décide de loger à la même pension où vivent celle qui s'appelle encore (pour le lecteur et pour le personnage-narrateur) Ana et celui qui semble (encore) être son père. Non sans ironie, il affirme: "No era la primera vez que me dejaba llevar más allá de la razón por un impulso libresco. Camino de casa me reí de mí mismo varias veces, pensando en Flaubert.» (Rey Rosa 2012:32). Il avoue ainsi à demi-mot avoir conscience de ressembler à un autre personnage de fiction, Emma Bovary, pensant sans doute à la très célèbre phrase de Flaubert, "Madame Bovary, c'est moi» alors que le lecteur (et peut-être lui-même) aurait vraisemblablement plutôt pensé que cétait Ana qui ressemblait à Emma. Et un peu plus tard, il remarquera qu'Ana signifie «Moi» en arabe (Rey Rosa 2012:71), comme si le narrateur et sa créature pouvaient se confondre parfois. Le personnage de fiction s'assume comme tel et sa condition de narrateur lui octroie une légitimité pour l'affirmer de façon ironique. Le libraire qu'est le personnage-narrateur est un grand lecteur: Madame Bovary, c'est lui, c'est nous. Dans une sorte de relation spéculaire, il se projette ironiquement comme lecteur dans la figure de la grande lectrice émotionnelle qu'est Emma Bovary alors que celle-ci s'identifie 
ingénument avec ce qu'elle lit, voulant vivre ce quelle lit avec passion. Emma désire ardemment que la littérature soit le fidèle reflet de sa réalité et Flaubert a su conférer à ses phrases et au discours indirect libre une ironie qui lui est propre afin de mettre en évidence cette posture d'Emma qui consiste à vouloir abolir la distance de la représentation, la coupure sémiotique. Et Rey Rosa a recours à une autre technique pour parvenir à créer un effet similaire et sans doute dupliqué.

Effectivement, la forme personnelle du narrateur de Severina introduit une ironie romantique singulière: le narrateur n'ignore pas son pouvoir de créer une réalité mais il connaît aussi ses limites (une réalité de langage) et les insinue au lecteur à travers de supposées intuitions. Dès la première ligne du roman, il affirme avoir suspecté Severina dêtre une voleuse de livres la première fois qu'il l'a vue alors même qu'elle n'avait rien volé ce jour-là. Puis il rêve de la mort d'Ana Severina et immédiatement il s'avère que celui qui est en train de mourir, c'est son père-grand-père-amant, qu'ils enterreront sur la crête d'un mont exactement comme dans le rêve qui se révèle être, rétrospectivement, une prémonition. Le narrateur devine presque toujours ce qu'est sur le point de dire Ana Severina, il a des pressentiments ou des présages, il pense qu'Ana Severina peut lire dans ses pensées comme dans un livre, etc., il est très intuitif, ce qui, bien entendu, est très ironique de la part d'un personnage narrateur puisque c'est lui qui raconte ce qu'il souhaite in fine. Et l'ironie est à son comble lorsque l'on se rappelle que l'on ne sait rien de Severina, pas même son nom. Cette "télépathie» amoureuse fait ainsi partie de l'ironie romantique du roman et le lecteur oscille entre la suspension de la crédulité et le sens critique face à cette relation si improbable entre une voleuse de livres sans identité et un libraire sans nom qui accepte le leurre au vu et au su de tous.

Il ne s'agit pas d'une véritable histoire d'amour, au sens d'une union s'installant dans la durée, mais de l'histoire d'une rencontre amoureuse durant laquelle la distance est abolie, le futur et le passé cessant d'exister pour laisser place à un présent pur. C'est le symptôme du présentisme. Ahmed, l'autre libraire, l’homme qui craint Dieu» (Rey Rosa 2012:43) est d'une autre époque comme semble l'indiquer sa librairie qui se trouve dans la rue Sucia de l'Ancienne ville de Guatemala tandis que celle du personnage-narrateur, La Entretenida (littéralement, "celle qui distrait» et, dans un second sens, la "maîtresse»), se trouve au sous-sol d'un centre commercial. Ahmed est le rival du narrateur amoureux d'Ana Severina, il tente de la garder à distance et ne la laisse pas voler de livres aussi facilement que le narrateur mais il n'ose pas dire à celui-ci qu'il lui a tout de même demandé sa main. À la fin du roman elle trompe Ahmed en lui offrant, pour réparer ses vols dans sa librairie, le supposé exemplaire du Coran volé dans la Bibliothèque de Borges, annoté par lui, d’une façon toute borgésienne si l'on y pense puisqu'il s'agit d'un «apocryphe». La distance critique des libraires a disparu. 


\section{Présentisme}

Létat de passion amoureuse qui se dessine dans Severina pourrait alors être comparé au présentisme, cette crise dans laquelle l'articulation passé-présentfutur est modifiée dans une tension nouvelle et aiguë entre le champ d'expérience et l'horizon d'attentes à tel point que la distance critique semble disparaître sous la menace d'un présent envahissant. Le narrateur avoue:

Muchas cosas pasaron, o, para ser más preciso, oí que pasaron muchas cosas por aquellos días (proliferaron los linchamientos en los pueblos del interior, hubo un golpe de estado en un país vecino, la coca ganó ventaja en la carrera global de las sustancias controladas, encontraron agua estancada en Marte, y Plutón perdió para siempre el status de planeta) porque mi vida había vuelto a reducirse a los libros, me había convertido en un ejemplar más de esa melancólica especie: el librero aspirante a escritor (Rey Rosa 2012:19-20).

Il révèle ainsi entre parenthèses que le monde extérieur existe, un monde rempli de sons et de fureur, mais qu'il souhaite oublier dans sa préoccupation intimiste pour les livres et sa nouvelle idylle. C'est presque un aveu de la part de Rodrigo Rey Rosa, écrivain qui nélude par ailleurs pas les thématiques historiques et politiques dans son œuvre si l'on se souvient de El material humano en particulier. Mais c'est surtout la confession du personnage-narrateur qui vit par et pour les livres, sous l'influence des livres et de la fiction. Et celle qui ravit tous les cœurs, en même temps que les livres, vient de réveiller chez lui, à ce moment de la diégèse, cet attrait mélancolique pour l'écriture. C'est tout l'univers de Severina qui converge vers les livres, comme si la crise de la représentation y avait subrepticement lieu et que le signe déplaçait la réalité fictive. Le narrateur aime penser, de façon borgésienne, que les frontières entre la fiction et la réalité sont poreuses; et ce n'est pas tout à fait absurde si l'on se souvient que certains livres volés par Ana Severina semblent annoncer certains éléments de l'intrigue - de façon ironique, notamment, avec Espérame en Siberia vida mía ou Viva México! Et le nom de la librairie, La Entretenida, semble nommer Severina, celle qui nous "distrait», nous «tient» en haleine. Les frontières entre la réalité et la fiction sont minces. Mais le lecteur est toujours déjà présent dans un rapport de représentation et Severina ne fait pas partie des livres volés de La Entretenida. Madame Bovary, c'est lui, et non pas elle, définitivement.

Cela peut éclairer d'un jour nouveau le personnage féminin de Severina. Nous ignorons ce que fait Severina avec les livres qu'elle vole, nous savons simplement qu'elle les lit avec son «grand-père» d'abord, puis avec le personnage-narrateur lorsqu'ils sont amants, dans la dernière partie du roman. Peut-être Severina estelle le narrataire de Severina. Son père-grand-père-amant, Otto, est l'idéologue d'une doctrine pour le moins excentrique sur l'empire des livres, son oncle croyait en la «lutte pour la domination livresque» avec une "guerre des classes de livres» (Rey Rosa 2012:57) et nous découvrons que toute l'ascendance de Severina vit et a vécu des livres, comme s'il s'agissait d'une religion ou d'une idéologie familiale. En volant des livres, Severina exprime à «sa manière» (Rey Rosa 2012:56) sa 
filiation, même floue et changeante. Et elle séduit le personnage-narrateur, qui la laisse voler de plus en plus de livres parce qu'il a l'impression qu'elle lui prête ainsi plus d'attention. Elle n'est pas Madame Blanco comme Emma pouvait être Madame Bovary, dont l'obsession tournait autour du statut social. Severina serait-elle une femme émancipée? Ce n’est pas si évident. Parce qu’Ana Severina est avant tout, aussi, une hystérique, elle a besoin d’être vue pour exister: lorsque le narrateur lui fait un compliment, elle ressemble tout à coup à une petite fille («una niñita después de hacer una proeza») (Rey Rosa 2012:26), la petite fille qui aimerait voler son père à sa mère, comme elle le fait d'une certaine façon avec son père-grand-père-amant. Ravir des livres, ravir des cœurs; cleptomanie et hystérie. Severina est certes théâtrale, comme toute bonne hystérique, il n’en reste pas moins quelle ne fait jamais de crise d'hystérie. Severina n'est pas un personnage étudié par Freud à la fin du XIX ${ }^{e}$ siècle, elle n’est pas Madame Bovary. Il n’y a pas de crise, pas de manière de sortir de la représentation, et peut-être pas non plus de la postmodernité.

\section{Conclusion}

Le roman Severina interroge non seulement les relations des livres à leurs lecteurs, à travers le personnage d'Ana Severina et de son grand-père, mais aussi les répercussions que peut avoir la représentation des livres dans la vie réelle et quotidienne. Le personnage-narrateur, qui n'a pas de nom à l'instar des auteurs des listes de livres volés, est un lecteur romantique, flaubertien, pour qui l'idéal absolu consiste à fusionner la vie et les livres. C'est, d'ailleurs, de toutes les références présentes dans le roman, Borges qui occupe la place centrale, maître indéniable et incontestable dans l'art littéraire de confondre le lecteur entre réalité et fiction. Ana Severina, à l'identité flottante et au passeport éternellement faux, est si invraisemblable qu'elle ne peut que nous faire penser à une métaphore de la littérature de Rey Rosa: universelle, antinationaliste, énigmatique et séduisante. Le roman ne prétend pas en finir avec la représentation qui entrerait dans une nouvelle crise mais il joue avec les fragments de la représentation réaliste, en miettes depuis déjà un certain temps.

D'une certaine manière, le personnage de Severina est symptomatique de notre époque: Severina souhaiterait exister en rompant avec la mémoire de ses ancêtres, elle aimerait se projeter dans un avenir stable, mais elle est condamnée à vivre des fragments de vies depuis un présent qui ne voit qu'une menace dans le futur. Et même ses crises de cleptomanie ne sont pas de véritables crises, elle ne fait qu’obéir aveuglément à une impulsion dictée par l'étrange doctrine de ses aïeux. Peut-être Severina vole-t-elle des livres pour être aimée. Sans doute prend-elle sa revanche sur les hommes, les libraires, cette espèce mélancolique que sont les «libraire[s] aspirant[s] écrivain[s]» (Rey Rosa 2012:20). Severina est-elle une victime des hommes, comme le fut à son époque, machiste, Emma Bovary sur le cadavre de laquelle "la masse des hommes de lettres du XIX ${ }^{\mathrm{e}}$ siècle discute»? (Nora Catelli 2001: 110). Est-elle un personnage n'existant que pour 
et par le regard des hommes - celui des personnages, des lecteurs, de lécrivain? $\mathrm{Ou}$, à présent que nous sommes plus majoritairement des lectrices, s'agit-il d'un personnage "féministe»? La seule certitude à ce sujet, c'est qu'il s'agit d'un personnage dont l'ambiguïté et l'indétermination activent l'interprétation du lecteur. Ou des lectrices.

\section{Bibliographie}

Barthes, R., 1970, S/Z, Paris, Seuil.

Bougnoux, D., 2006, La crise de la représentation, Paris, La Découverte.

Catelli, N., 2001, Testimonios tangibles, Barcelona, Anagrama.

Derrida, J., 1972, «La différance», in: Marges de la philosophie, Les éditions de minuit, Paris.

Ezquerro, M., 2002, Fragments sur le texte, Paris, L'Harmattan.

Flaubert, G., 2001, Madame Bovary, Paris, Gallimard.

Hartog, F., 2012, Régimes d'historicité, Paris, Seuil.

Iser, W., 1976, L'acte de lecture. Théorie de l'effet esthétique, traduit de l'allemand par Evelyne Sznycer, Bruxelles, Pierre Mardaga.

Jauss, H. R., 1978, Pour une esthétique de la réception, traduit de l'allemand par Claude Maillard, Paris, Gallimard.

Lyotard, J.-F., 1979, La condition postmoderne, Paris, Les éditions de minuit.

Poulet, G., 1979, Les métamorphoses du cercle, Paris, Flammarion.

Rey Rosa, R., 2012, Severina, Buenos Aires, Alfaguara.

Salmon, Ch., 2012, Le storytelling: la machine à fabriquer des histoires et à formater les esprits, Paris, La Découverte. 